\title{
STOCHASTIC CARBON EMISSION ESTIMATION METHOD FOR CONSTRUCTION OPERATION
}

\author{
Chang-Yong YI, Han-Seong GWAK, Dong-Eun LEE \\ School of Architecture and Civil Engineering, Kyungpook National University, 1370. \\ Sangyegk-Dong, Buk-Gu, DaeGu, 702-701, Korea
}

Received 24 Mar 2014; accepted 15 Aug 2014

\begin{abstract}
Low carbon construction is an important operation management goal because greenhouse gas (GHG) reduction has become a global concern. Major construction resources that contribute GHG, such as equipment and labour, are being targeted to achieve this goal. The GHG emissions produced by the resources vary with their operating conditions. It is commendable to provide a statistical GHG emission estimation method that models the transitory nature of resource states at micro-scale of construction operations. This paper proposes a computational method called Stochastic Carbon Emission Estimation $\left(\mathrm{SCE}^{2}\right)$ that measures the variability of $\mathrm{GHG}$ emissions. It creates construction operation models consisting of atomic work tasks, utilizes hourly equipment fuel consumption and hourly labourer respiratory rates that change according to their operating conditions classified into five categories, and identifies an optimal resource combination by trading off eco-economic performance metrics such as the amount of GHG emissions, operation completion time, operation completion cost, and productivity. The study is of value to researchers because $\mathrm{SCE}^{2}$ fill in a gap to eco-economic operation modelling and analysis tool which considers operating conditions at micro-scale of construction operation having many stochastic work tasks. This study is also relevance to practitioners because it allows project managers to achieve eco-economic goals while honouring predefined constraints associated with time and cost.
\end{abstract}

Keywords: greenhouse gas emission, environmental impact, simulation, construction operation model, eco-economics.

\section{Introduction}

The construction industry is one of the greatest consumers of raw materials and resources and is responsible for more than $30 \%$ of total carbon emissions (Tae et al. 2011). Since the industry plays such a major role in global environment degradation, controlling and reducing GHG emissions has become one of its major tasks (Liu et al. 2013). Existing research aimed at reducing GHG has primarily focused on estimating the amount of emissions using life cycle assessment (LCA). However, few have investigated the construction stage in particular (Yan et al. 2010). Note is the fact that the method measuring the environmental impacts from the construction phase not only completes the analysis of total life cycle environmental impact, but also provides the construction community with a new opportunity to minimize environmental impacts.

Equipment use is the main contributor to environmental impacts in the construction phase. A $10 \%$ reduction in diesel fuel use would reduce construction sector $\mathrm{CO}_{2}$ emissions by approximately 5\% (EPA 2009). Thus, a computational method that estimates the amount of GHG emissions attributable to equipment intensive operations provides an important measure to establish an eco-economic construction plan. The amount of GHG emissions generated by equipment over the duration of activity varies because the amount of fuel consumed changes depending on the operating condition (i.e., haul road surface condition, altitude, and temperature) and the load factor (Peurifoy et al. 2009). The equipment attributes involved in GHG emission computation (i.e., the type of equipment, hourly fuel consumption in a particular operating condition, and hourly operating cost) (Caterpillar 2010) and the labourer's respiratory rates per working condition (i.e., the number of breaths, inhalation and exhalation cycles, per time unit) (Sherwood 2006) provide important sources for estimating the amount of GHG emitted by construction resources. Given that this information is integrated with a discrete event simulation (DES)-based construction operation model, it may provide more reliable statistical results on activity estimates for micro-scale equipment operation and emission estimates. DES is definitely well accepted as a useful modelling and analysis method that handles the variability of GHG emission of construction resources assigned to a construction operation and represents properly the project uncertainties (Ahn et al. 2010; Gonzalez, Echaveguren 2012). The existing methods are well established

Corresponding author: Dong-Eun Lee

E-mail:dolee@knu.ac.kr 
and available in theory and application. However, it would be desirable to offer a computational method that takes into account the transitory nature of the resource state that changes at a particular point of simulation time, combines the equipment and labourer data sources that administrate their GHG emission with DES, and integrates an operation model obtained from an external DES system directly, and estimates the variability of the eco-economic metrics of the operation (i.e., carbon emission amount, operation completion time, and operation completion cost) by providing an automated easy-to-use interface.

This paper presents a stochastic carbon emission estimation $\left(\mathrm{SCE}^{2}\right)$ method which measures the variability of GHG emissions. SCE ${ }^{2}$ allows modelling the transitory nature of the resource states (i.e., operating conditions) at micro-scale of construction operation having many stochastic work tasks. It provides an eco-economic operation modelling and analysis tool that improves the accuracy of carbon emission estimation and allows practitioners to make more informed decisions achieving eco-economic construction plans.

This paper is organized as follows. First, the author(s) examine existing GHG emission estimation methods, identify the major factors limiting their performance, and discuss various strategies for eliminating these limitations. Second, the variables influencing the amount of carbon emissions are identified and mathematical formulas for computing amount of GHG emissions are developed using them. Third, the proposed computational method is coded into the system called the stochastic carbon emission estimation method ( $\mathrm{SCE}^{2}$ ) based on MATLAB (Ver. 2008b) to address real-world cases frequently encountered in practice and provide practitioners and researchers with an easy-to-use interface. It estimates the variability of eco-economic metrics of construction operations using DES. Finally, the authors provides a detailed illustration of the computational method and verify the performance of $\mathrm{SCE}^{2}$ using a real-world earthmoving operation scenario (Halpin, Riggs 1992).

\section{Current state of environmental impact assessment research in the construction industry}

Given the interaction complexities between construction processes and natural environments, LCA provides a science-based, fairly comprehensive, and standardized assessment approach to quantify the environmental impacts of a construction project over its entire life cycle (Zapata, Gambatese 2005; Vieira 2007; Ragheb 2011). However, most of the current LCA tools geared towards the entire life cycle of a project improperly address the environmental impact of construction processes. Only a few research efforts have ever attempted to assess the environmental impacts of construction processes and/or operation (Sharrard et al. 2008; Cass, Mukherjee 2011; Li et al. 2010; Ahn, Lee 2012).
The only available data that can be used to quantify the environmental impact of equipment in most LCA research on construction processes is the daily report on how many pieces of equipment are deployed on a job site. However, this data source is lacking in detailed information regarding the efficiency of equipment usage and equipment hours. In order to calculate GHG emissions using existing life cycle impact assessment metrics and methods, historical performance data should be organized along with accurate construction inventories (i.e., materials installed and equipment used in construction and maintenance operations) (Cass, Mukherjee 2011; Ahn, Lee 2012).

A few researchers have proposed DES-based methods that assess the environmental impacts of construction operations to complement the existing LCA based methods for tunnelling operation (Ahn et al. 2010), earthmoving operation (Ahn, Lee 2012), road construction (Gonzalez, Echaveguren 2012), and crane selection (Hasan et al. 2013). They built upon the foundation that optimizing equipment operations in the construction operation planning phase effectively reduces the environmental impact. They contributed to the identification of alternative resource assignment plans for equipment intensive operations by handling a situation in which the amount carbon emission changes according to equipment states (i.e., active or idle).

In the initial modeling phase, care should be taken to ensure that resource states are properly classified in an appropriate detail and defined in the model. The existing systems allow only two states, i.e., idle state in queue and active state in a work task. However, a resource entity may have various resource states in different work tasks. The new method improves the accuracy of existing methods by providing easy-to-use tool to define the resource states into the full range of possible states (i.e., 5 categories) and to modify the average hourly fuel consumption in each and every state. In addition, it provides a multiobjective optimization method for searching the optimal resource combination of a construction operation.

A new computational method that implements eco-economic modelling and analysis for construction operations is proposed in this paper. The method can create an operation model or import operation templates from existing DES systems. Further, it identifies optimal resource combinations such as equipment fleet and labour crew that minimize operation completion carbon emission $\left(\mathrm{OCCO}_{2}\right)$, operation completion time (OCT), and/or operation completion cost (OCC). The method is implemented in software called Stochastic Carbon Emission Estimation $\left(\mathrm{SCE}^{2}\right)$ method. $\mathrm{SCE}^{2}$ estimates the best-fit-PDFs of the eco-economic metrics without user intervention at any time. It also encourages the use of eco-economic metrics in decision making involved in project goals by querying the probability of completing the project within specific boundary values of eco-economic metrics (i.e., $\mathrm{OCCO}_{2}, \mathrm{OCT}$, and $\mathrm{OCC}$ ). 


\section{Stochastic carbon emission estimation method for construction operations}

The method that leads to carbon emission reduction by identifying optimal resource combination and which represents the algorithm used in $\mathrm{SCE}^{2}$ is presented in Figure 1. The method described below was coded into an automated system that imports an operation model from an existing DES system, sets resource attributes, and executes the DES by using MATLAB to improve the usability of the computational method in eco-economic considerate operation planning practice.

\subsection{Creating Eco-economic Considerate Construction Operations}

As shown in Figure 1, $\mathrm{SCE}^{2}$ creates an operation model using an existing DES system (i.e., COPS (Lee et al. 2010)), reads the operation model data (i.e., operation ID, operation name, length of simulation, maximum number of cycles, a matrix of component ID and name, predecessors and successor vectors, and the primary keys of which resources are assigned to each work assignment) and saves these information in computer memory for simulation runs in Step 1. The $\mathrm{SCE}^{2}$ method introduces the primary keys of task components of the operation model (i.e., the component ID) as foreign keys to establish a relationship between task components in a "components" table and work assignments in a "resources" table, as shown in Table 1. The relationship between task component and work assignment is one-to-many (1:M), because more than one work assignment having different assignment ID (i.e., $W_{I D}$ ) can be associated with a work task (i.e., component ID). For example, two work assignments (e.g., such as $W_{I D}=7$ and 8) are individually given to two different resource entities (e.g., Wheel loader: 990SII and Truck 773D) that work together to perform a work task (e.g., Load truck; task component ID $=7$ ). The relationships are defined by the user.

\subsection{Assigning resource entities to work tasks}

$\mathrm{SCE}^{2}$ implements two types of queue components: resource entities and idle queues. The resource entities are initialized at the corresponding resource queues by setting the resource $I D\left(R_{I D}\right)$, the type of resource $\left(R_{T Y}\right)$,

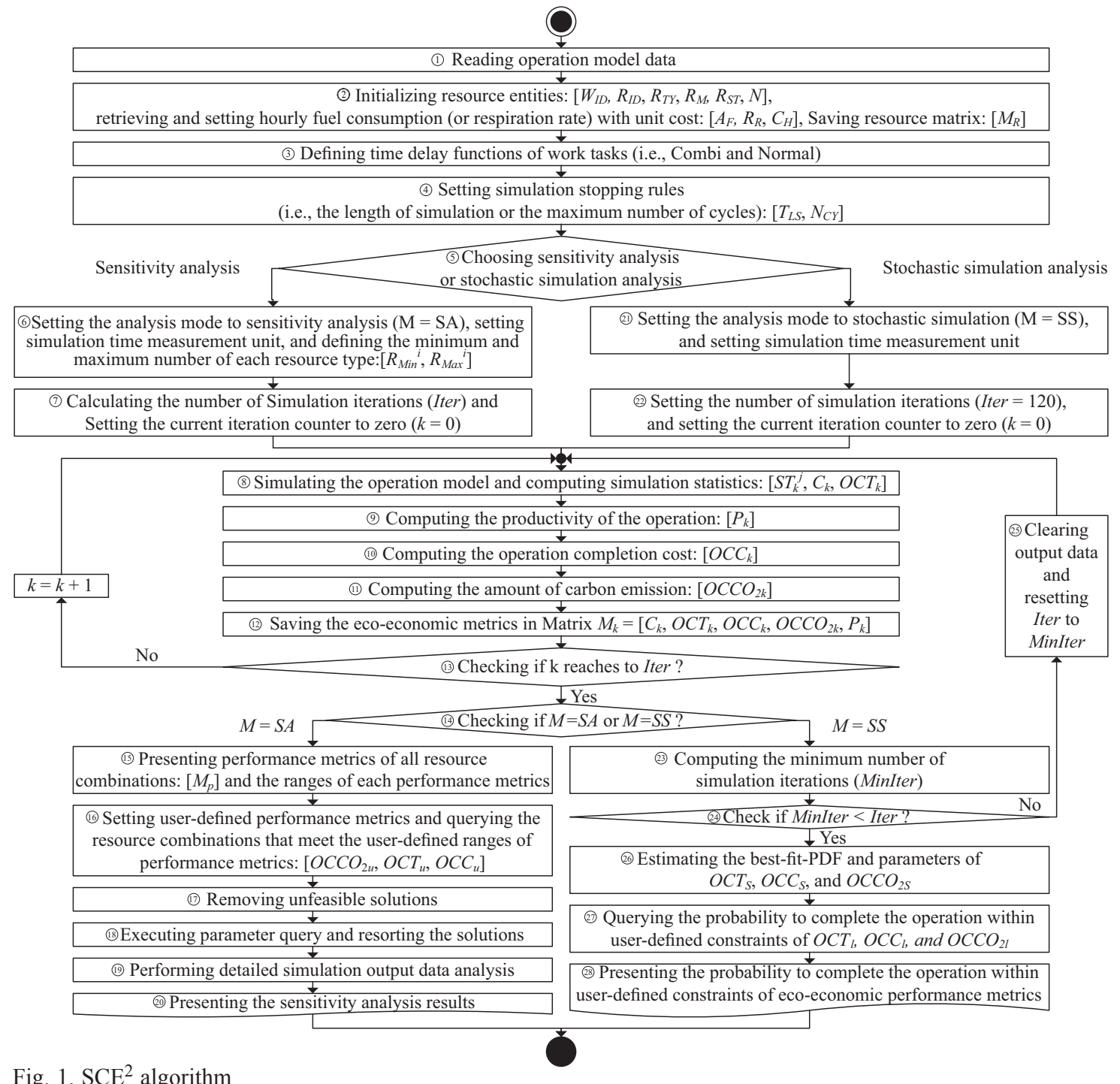

Fig. 1. $\mathrm{SCE}^{2}$ algorithm 


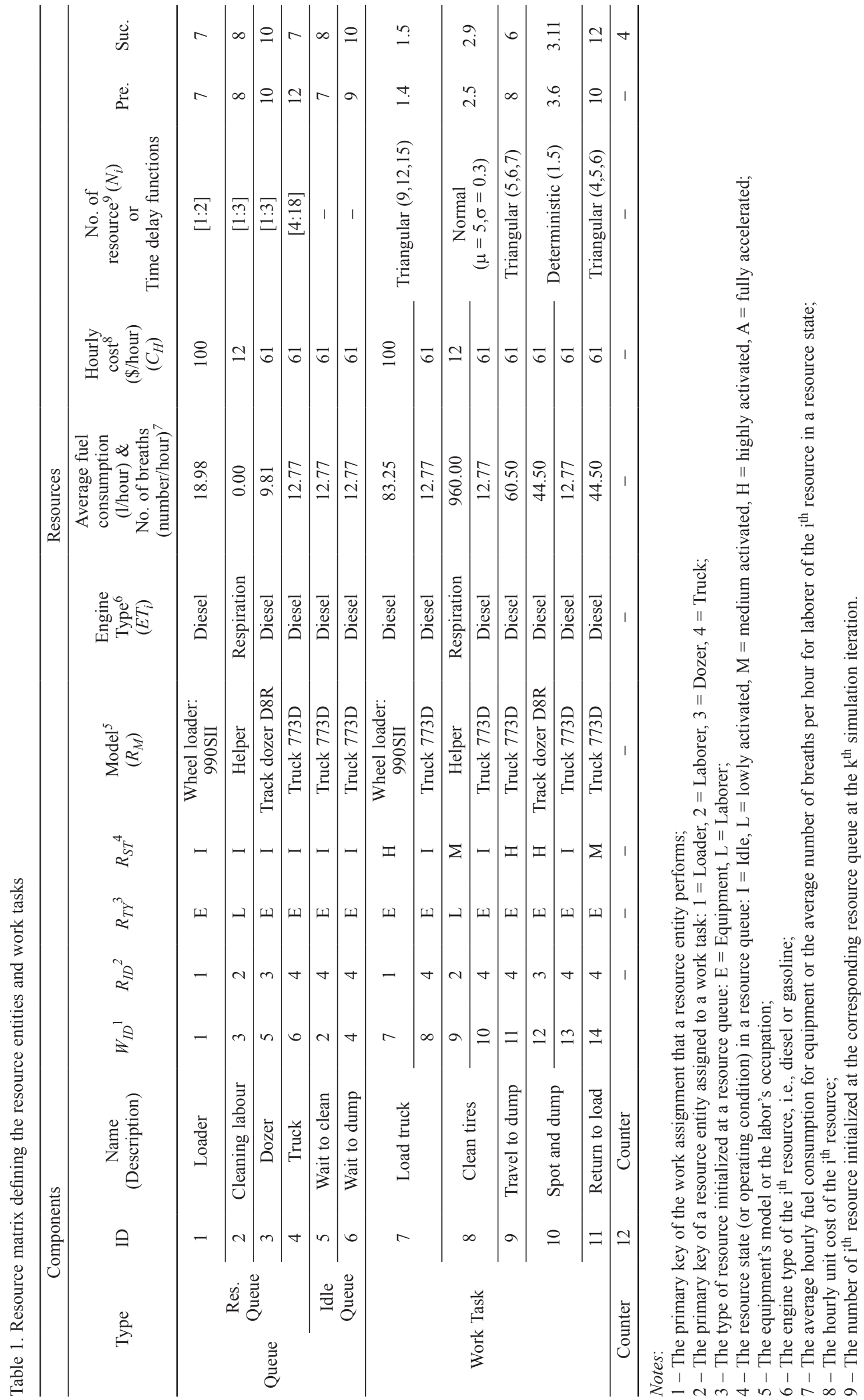


the equipment's model or the labourer's occupation $\left(R_{M}\right)$, and the number of resources $\left(N_{i}\right)$ to be initialized, as shown in Table 1. It specifies the resource state $\left(R_{S T}\right)$ of each resource entity in the corresponding resource queue as "idle state ( $I$ )" by default because the resource entities wait in the queue before starting an operation. Given the resource $I D \mathrm{~s}\left(R_{I D}\right)$ of the resource entities to be captured in each idle queue at simulation run time, it also specifies the resource state $\left(R_{S T}\right)$ of the corresponding resource entity as "idle state $(I)$ " without user involvement at simulation run time by default.

\subsection{Retrieving resource attributes involved in GHG emission computation}

After defining the resource states $\left(R_{S T}\right), \mathrm{SCE}^{2}$ creates a query in structured query language (SQL) using resource matrix $M_{R}$, queries an external resource database (which is presented as a partial table owing to lack of space in Table 2), retrieves equipment hourly average fuel consumption $\left(A_{F}\right)$ and/or the labourers' respiratory rate $\left(R_{R}\right)$ with their hourly unit costs $\left(C_{H}\right)$ from the corresponding databases. It retrieves these pieces of information using the resource entities' attributes (i.e., gasoline or diesel engine type $\left(E_{T Y}\right)$ for equipment or a fixed respiratory rate for labourers) and the resource state $\left(R_{S T}\right)$ (i.e., idle $(I)$, lowly activated $(L)$, medium activated $(M)$, highly activated $(H)$, or accelerated $(A))$ from the corresponding database. For example, given that $W_{I D}, R_{I D}, R_{T Y}$, and $R_{S T}$ are 1, 1, E, and I, respectively, the method executes the SQL statement shown in Eqn (1). Then, it saves this information in resource matrix $M_{R}$ that corresponds to columns 5 to 12 of Table 1 in Step 2:

$$
\begin{aligned}
& \text { Insert into Table } 1\left(A_{F}, C_{H}\right) \\
& \text { Select } A_{F}, C_{H} \text { FROM Table } 2 \\
& \text { Where ' } R_{I D}=1 \text { ' and ' } R_{T Y}=\mathrm{E} \text { ' and ' } R_{S T}=\mathrm{I} \text {, }
\end{aligned}
$$

where: $A_{F}$ is the average hourly fuel consumption or the average number of breaths of a resource according to the resource state; $C_{H}$ is the hourly unit cost of a resource; $R_{I D}$ is the primary key of a resource of which a resource entity is assigned to a component; $R_{T Y}$ is the type of resource defining which type of resource entities (i.e., $\mathrm{E}=$ Equipment, $\mathrm{L}=$ Labourer) are initialized at each resource queue; $R_{S T}$ is the resource's state (or operating condition) involved in the operating condition in a component: idle (I), lowly activated (L), medium activated (M), highly activated $(\mathrm{H})$, and fully accelerated.

\subsection{Specifying operating conditions}

The resource state $\left(R_{S T}\right)$ corresponds to the operating state of the equipment engine. It is represented by the load factor, which depends on the operating condition (e.g., haul distance, road's maintenance condition, grade resistance, and rolling resistance). It reaches $100 \%$ when the engine develops its maximum horsepower (HP) continuously, and decreases when the engine waits in the idle queue, moves while empty, or moves down a declined slope. In this paper, load factors are assumed to be $10 \%$ when the engine is idling, $20-30 \%$ at low horsepower (HP), $30-40 \%$ at medium, $40-50 \%$ at high, and $100 \%$ at fully accelerated horsepower (HP). These are either directly adapted from three engine operating states (i.e., low, medium, and high) or extrapolated from the load factors of the idle and accelerated states (Caterpillar 2010). The ranges of hourly fuel consumptions of these five statuses are computed by assuming that load factor is proportional to the hourly fuel consumption and is used to compute the amount of carbon emission, as shown in Table 3 (Caterpillar 2010).

Table 3. Fuel consumption of articulated trucks according to the load factor (adapted from Caterpillar 2010, Unit: 1/hour)

\begin{tabular}{cccc}
\hline Model & \multicolumn{3}{c}{ Load factors } \\
\cline { 2 - 4 } $\begin{array}{c}\text { Articulated } \\
\text { Trucks }\end{array}$ & Low* & Medium** & High*** \\
\hline D25D & $13.1-18.3$ & $18.3-25.7$ & $25.7-37.1$ \\
\hline D30D & $14.7-20.4$ & $20.4-28.7$ & $28.7-41.5$ \\
\hline D250E & $13.2-18.3$ & $18.3-25.8$ & $25.8-37.2$ \\
\hline
\end{tabular}

Notes:

* Low: Short to medium hauls on well-maintained level haul roads. Minimum total resistance;

** Medium: Normal load and haul time. Varying load and haul road conditions. Some adverse grades. Some high rolling resistance;

*** High: Long haul time with frequent adverse grades. Continuous use on very poorly maintained haul roads with high rolling resistance.

A labourer's respiratory rate while idle (e.g., resting), active with low working load (e.g., driving), active with medium working load (e.g., cycling), active with high working load (e.g., mounting), and active in fully accelerated working load (e.g., shallow and rapid breathing) states are 12, 18, 28, 34, and 40 breaths per minute,

\begin{tabular}{|c|c|c|c|c|c|c|c|c|c|}
\hline \multirow[t]{2}{*}{ Database } & \multirow{2}{*}{$\begin{array}{l}\text { Resource } \\
\text { ID }\left(R_{I D}\right)\end{array}$} & \multirow{2}{*}{$\begin{array}{c}\text { Model } \\
\text { (or Occupation) } \\
\left(R_{M}\right)\end{array}$} & \multirow{2}{*}{$\begin{array}{l}\text { Engine type } \\
\quad(\mathrm{ET})\end{array}$} & \multicolumn{5}{|c|}{$\begin{array}{c}\text { Average Fuel Consumption (1/hour) \& } \\
\text { Respiration (number/hour) }\left(A_{F}\right)\end{array}$} & \multirow{2}{*}{$\begin{array}{c}\text { Hourly } \\
\text { Cost }(\$ / \text { hour })\left(C_{H}\right)\end{array}$} \\
\hline & & & & Idle & Low & Medium & High & Accelerate & \\
\hline \multirow{3}{*}{ Equipment } & 1 & Wheel Loader: 990SII & Diesel & 18.98 & 49.00 & 64.25 & 83.25 & 189.79 & 100 \\
\hline & 3 & Track Type Dozer: D8R & Diesel & 9.81 & 25.50 & 33.00 & 44.50 & 98.14 & 61 \\
\hline & 4 & $\begin{array}{c}\text { Construction Truck: } \\
\text { 773D }\end{array}$ & Diesel & 12.77 & 30.25 & 44.50 & 60.50 & 127.72 & 61 \\
\hline Labour & 2 & Labourer: Helper & Respiration & 0 & 360 & 960 & 1320 & 1680 & 12 \\
\hline
\end{tabular}
respectively (Sherwood 2006; Int Panis et al. 2010).

Table 2. Resources database 
The average number of breaths per minute is used to compute the volume of air exhaled from a healthy adult male's lungs in one minute. The amount of carbon exhaled by a worker is estimated using this volume. Note that the amount of carbon produced by the human body's metabolism in daily life should be excluded from the figure in order to get the net carbon emission attributed to construction activities only. For this reason, the labourer's hourly respiratory rates while idle, active with low working load, active with medium working load, active with high working load, and active with fully accelerated working load states are $0(=12-12), 360(=(18-12) \times$ $60), 960(=(28-12) \times 60), 1320(=(34-12) \times 60)$, and $1680(=(40-12) \times 60)$ breaths per hour, respectively.

\subsection{Defining time delay functions of work tasks and stopping rules}

The time delay functions of all work tasks (i.e., COMBI or NORMAL) are defined in particular probability distribution functions (PDFs) with their parameters in Step 3. They are estimated by using the automated best-fit-PDF algorithm championed by Ang and Tang (1975) and Lee et al. (2010) without user involvement if historical task durations are available. The variates of task durations are generated using a random number generator (such as $\operatorname{rand}(0)$ function) that operates within the confines of the PDF of the time delay function and their parameters. After defining the time delay function of a work task, the type of resource $\left(R_{T Y}\right)$ and the resource state $\left(R_{S T}\right)$ involved in the work task are defined by the user. For example, when a loader (e.g., Wheel loader 990SII) and a truck (e.g., Truck 773D) are assigned to perform the "Load truck" work task, assignments 7 and 8 are performed by Wheel loader 990SII and Truck 773D, respectively. The resource states of the loader and the truck are active working and idle states, respectively, when they are captured by "Load truck" work task. The resource state $\left(R_{S T}\right)$ of each resource captured by a work task is defined by considering the operating conditions given in the load factor guide provided in Caterpillar's performance handbook (Caterpillar Inc. 2010).

$\mathrm{SCE}^{2}$ sets two stopping rules, the maximum number of cycles $\left(N_{C Y}\right)$ and the length of simulation $\left(T_{L S}\right)$, to decide when to terminate the simulation. The former terminates the simulation experiment when the production unit (e.g., truck loaded with earth at full capacity) reaches a predefined maximum number of cycles (e.g., 30 cycles). The latter terminates the simulation experiment when the elapsed simulation time $\left(T_{S}\right)$ is equal to the length of the simulation (e.g., 10,000 seconds). These data members of the counter component are defined to these values (i.e., $\left.N_{C Y}, T_{L S}\right)$ at simulation design time in Step 4.

\subsection{Simulating operation model with sensitivity analysis}

$\mathrm{SCE}^{2}$ provides sensitivity analysis that evaluates the ecoeconomics performance metrics $\mathrm{OCCO}_{2}, \mathrm{OCT}$, and $\mathrm{OCC}$ given all resource combinations and stochastic simulation analysis that computes the variability of the eco-economics performance metrics given a specific resource combination in Step 5. Sensitivity analysis definitely changes the resource combination (i.e., the number of resources) assigned to the operation model in each simulation iteration, whereas stochastic simulation analysis keeps the optimal resource combination during the simulation experiment, but runs the simulation experiment many times.

The sensitivity analysis method starts by setting the analysis method variable to sensitivity analysis (i.e., $M=S A)$. The time measurement unit of the simulation clock (i.e., second, minute, or hour) is defined to make the unit of time used by the equipment's fuel consumption (or labourer's respiration measurement) the same as that of the time delay functions of the work tasks right before executing simulation experiments. The minimum and maximum numbers of $i^{\text {th }}$ resource types (i.e., $R_{\min }{ }^{i}, R_{\max }{ }^{i}$ ) are defined in a certain range in Step 6. The number of simulations (Iter) is computed using the two point estimates $\left(R_{\min }{ }^{i}, R_{\max }{ }^{i}\right)$ as shown in Eqn (2). The current iteration counter is set to zero $(k=0)$ in Step 7. For example, if four different types of resource (i.e., count $\left.\left(R_{I D}\right)==4\right)$ are initialized at four different resource queues and the minimum and maximum numbers of $1^{\text {st }}$, $2^{\text {nd }}, 3^{\text {rd }}$, and $4^{\text {th }}$ resources are 1 to 2,1 to 3,1 to 3 , and 4 to 18 , the total number of resource combinations is 270 $(=2 \times 3 \times 3 \times(18-4+1))$. Therefore, the number of simulations (Iter) would be 270 in this case:

$$
\text { Iter }=\prod_{i=1}^{\operatorname{count}\left(R_{I D}\right)}\left(R_{\text {max }}^{i}-R_{\text {min }}^{i}+1\right),
$$

where: Iter is the initial number of simulations; $R_{\min }{ }^{i}$ and $R_{\max }{ }^{i}$ are the minimum and maximum number of the $i^{\text {th }}$ resource type.

Simulation experiments are executed for all possible resource combinations in an enumerative manner to determine the global optimal resource combination that produces the best eco-economic performance metrics. The $\mathrm{SCE}^{2}$ method computes the resource entity's average staying time at the $j^{\text {th }}$ component (i.e., average idle time at a queue component (QUEUE), and average service time at a work task component (i.e., COMBI and NORMAL)) in the $k^{\text {th }}$ simulation iteration $\left(S T_{k}^{j}\right)$, the number of completed cycles going through the counter component (COUNTER) at the $k^{\text {th }}$ simulation iteration $\left(C_{k}\right)$, and the elapsed simulation time when the simulation terminates at the $k^{\text {th }}$ simulation iteration $\left(O C T_{k}\right)$ according to Kelton and Law (2000) in Step 8. If the simulation terminates right after the $T_{L S}$ elapses, the $O C T$ is the same as the $T_{L S}$; if the simulation terminates right after the maximum number of cycles $\left(N_{C Y}\right)$ has been completed, the time when the last production unit is captured by counter component $\left(T_{N}\right)$ would be the operation completion time $\left(O C T_{k}\right)$ at the $k^{\text {th }}$ simulation iteration, as defined by Eqn (3):

$O C T=\left\{\begin{array}{l}T_{L S} \text { if stopping rule }=\text { the number of cycles } \\ T_{S} \text { if stopping rule }=\text { the simulation time }\end{array}\right.$, 
where: $O C T$ is the elapsed simulation time when the simulation terminates; $T_{L S}$ is the amount of simulation time, which terminates the simulation when the simulation system clock time has elapsed; $T_{S}$ is the simulation system clock time.

The productivity of the operation at the $k^{\text {th }}$ simulation iteration $\left(P_{k}\right)$ is computed using the number of completed cycles $\left(C_{k}\right)$ and the elapsed simulation time when the simulation stopped at the $k^{\text {th }}$ simulation iteration $\left(O C T_{k}\right)$ using Eqn (4) in Step 9:

$$
P_{k}=\frac{C_{k}}{O C T_{k}},
$$

where: $P_{k}$ is the productivity of the operation at the $k^{\text {th }}$ simulation iteration; $C_{k}$ is the number of cycles reached by a counter component at the $k^{\text {th }}$ simulation run; $O C T_{k}$ is the elapsed simulation time when the simulation terminates at the $k^{\text {th }}$ simulation iteration.

The $O C C$ at the $k^{\text {th }}$ simulation iteration $\left(O C C_{k}\right)$ is computed using the number of $i^{\text {th }}$ resource type assigned to the operation model $\left(N_{k}^{i}\right)$, the $i^{\text {th }}$ resource's hourly costs $\left(C_{H}^{i}\right)$, and the elapsed simulation time $\left(O C T_{k}\right)$ at the $k^{\text {th }}$ simulation iteration using Eqn (5) in Step 10:

$$
\text { OCC }_{k}=\sum_{i=1}^{\operatorname{count}\left(R_{I D}\right)} C_{H}^{i} \times N_{k}^{i} \times O C T_{k},
$$

where: $O C C_{K}$ is the operation completion cost at the $k^{\text {th }}$ simulation iteration; $C_{H}{ }^{i}$ is the hourly unit cost of the $i^{\text {th }}$ resource; $N_{k}^{i}$ is the number of $i^{\text {th }}$ resources initialized to the corresponding resource components at the $k^{\text {th }}$ simulation iteration.

The computational method checks if the $i^{\text {th }}$ resource type is equipment or labourer. If it is equipment, the method determines its ton of oil equivalent (TOE, $O_{i}$ ) and carbon emission factor $\left(C E F_{i}\right)$ by referring to IPCC (2006) and depending on the engine type of the $i^{\text {th }}$ resource $\left(E T_{i}\right)$, as shown in Eqns (6) and (7). The TOEs of diesel and gasoline engines are 0.000845 (toe) and 0.000745 (toe), respectively. The carbon emission factors $\left(C E F_{i}\right)$ of diesel and gasoline engines are 0.837 (toe-C/ ton) and 0.783 (toe-C/ton), respectively.

$$
\begin{aligned}
& O_{i}=\left\{\begin{array}{ll}
0.000845 ; & \text { if } E T_{i}=\text { diesel } \\
0.000740 ; & \text { if } E T_{i}=\text { gasoline }
\end{array} ;\right. \\
& C E F_{i}=\left\{\begin{array}{ll}
0.837 ; & \text { if } E T_{i}=\text { diesel } \\
0.783 ; & \text { if } E T_{i}=\text { gasoline }
\end{array},\right.
\end{aligned}
$$

where: $O_{i}$ is ton of oil equivalent (TOE) of the $i^{\text {th }}$ resource; $E T_{i}$ is the engine type of the $i^{\text {th }}$ resource; $C E F_{i}$ is the carbon emission factor of the $i^{\text {th }}$ resource, provided by IPCC (2006).

The carbon emission amount $\left(\mathrm{kg} C \mathrm{O}_{2 k}^{i}\right)$ is computed using the respiratory volume (i.e., $0.51 /$ man-breath), and its corresponding carbon dioxide rate (3.97\%, i.e., $0.03 \%$ for inhalation and $4 \%$ for exhalation) in a man-breath volume when the $i^{\text {th }}$ resource type is labourer. The con- stant $\frac{44}{22.4}$ represents the theoretical molecular weight of $\mathrm{CO}_{2}$ (i.e., $44 \mathrm{~g} / \mathrm{mol}$ ), which means that $44 \mathrm{~g}$ of $\mathrm{CO}_{2}$ is generated for every 22.41 of air a person breathes (Sherwood 2006).

The total amount of carbon emission generated by the $i^{\text {th }}$ resource $\left(\mathrm{kgCO} i{ }_{2 k}^{i}\right)$ is computed using the average hourly fuel consumption ( $\left.E C_{i}^{j}\right)$ or the average hourly respiratory rate that changes according to the $i^{\text {th }}$ resource's state $\left(R_{S T i}^{j}\right)$ captured to the $j^{\text {th }}$ component, the resource entity's average staying time ( $\left.S T_{k}^{j}\right)$ at the $j^{\text {th }}$ component, the number of entities arriving at the $j^{\text {th }}$ component in the $k^{\text {th }}$ simulation iteration $\left(N E_{k}^{j}\right)$, as shown in Eqn (8). The constants 1000 and 0.001 are used to unify the unit of carbon emission to kilogram $(\mathrm{kg})$ :

$$
\operatorname{kgCO} O_{2 k}^{i}=\left\{\begin{array}{c}
\sum_{j=1}^{\operatorname{count}\left(W_{I D}\right)} E C_{i}^{j} \times S T_{k}^{j} \times N E_{k}^{j} \times O_{i} \times C E F_{i} \times \frac{44}{12} \times 1000 \\
\text { if } E T_{i}=\text { diesel or gasoline, and } \forall i \in I R_{j} \\
\operatorname{count}\left(W_{I D}\right) E C_{i}^{j} \times S T_{k}^{j} \times N E_{k}^{j} \times 0.5 \times 0.0397 \times \frac{44}{22.4} \times 0.001 \\
\sum_{j=1} \quad \text { if } E T_{i}=\text { respiration, and } \forall i \in I R_{j},
\end{array}\right.
$$

where: $\mathrm{kgCO}_{2 k}^{i}$ is the amount of carbon emission of the $i^{\text {th }}$ resource at the $k^{\text {th }}$ simulation run; $E C_{i}^{j}$ is the average hourly fuel consumption or respiratory rate of the $i^{\text {th }}$ resource at the $j^{\text {th }}$ component; $S T_{k}^{j}$ is the average service (or idle, staying) time of the $j^{\text {th }}$ work task component at the $k^{\text {th }}$ simulation iteration; $N E_{k}^{j}$ is the number of entities that have arrived at the $j^{\text {th }}$ component in the $k^{\text {th }}$ simulation iteration.

The average hourly fuel consumption ( $E C_{i}^{j}$ ) or the average hourly respiratory rate, which changes depending on the $i^{\text {th }}$ resource's state $\left(R_{S T i}^{j}\right)$ captured to the $j^{\text {th }}$ component, is computed using Eqn (9):

$$
E C_{i}^{j}\left\{\begin{array}{l}
A_{F}\left(I_{i}\right) ; \text { if } R_{S T i}^{j}=\text { Idle } \\
A_{F}\left(L_{i}\right) ; \text { if } R_{S T i}^{j}=\text { Low } \\
A_{F}\left(M_{i}\right) ; \text { if } R_{S T i}^{j}=\text { Medium } \\
A_{F}\left(H_{i}\right) ; \text { if } R_{S T i}^{j}=\text { High } \\
A_{F}\left(A_{i}\right) ; \text { if } R_{S T i}^{j}=\text { Acceleration }
\end{array}\right.
$$

where: $A_{F}\left(I_{i}, L_{i}, M_{i}, H_{i}, A_{i}\right)$ is the average hourly fuel consumption or the average number of breaths of the $i^{\text {th }}$ resource in the idle $(I)$, low $(L)$, medium $(M)$, highly activated $(H)$, and fully accelerated $(A)$ resource state, respectively; $R_{S T i}^{j}$ is the $i^{\text {th }}$ resource's state (or operating condition) involved in the operating condition in the $j^{\text {th }}$ component.

The total amount of carbon emission produced by the operation in the $k^{\text {th }}$ simulation iteration $\left(\mathrm{OCCO}_{2 k}\right)$ is computed by summing the amounts of carbon emission produced by all resource entities assigned to the operation $\left(k g C O_{2 k}^{i}\right)$, as shown in Eqn (10): 


$$
\mathrm{OCCO}_{2_{k}}=\sum_{i=1}^{\operatorname{count}\left(R_{I D}\right)} k g C O_{2 k}^{i},
$$

where: $O C C O_{2 k}$ is the amount of carbon emission produced by the operation at the $k^{\text {th }}$ simulation run.

\subsection{Computing the variability of eco-economic performance metrics}

The eco-economic performance metrics, $C_{k}, O C T_{k}$, $O C C_{k}, O C C O_{2 k}$, and $P_{k}$, computed in each iteration of the simulation are saved to computer memory in Step 12. The method saves the eco-economic performance metrics, $C_{s}, O C T_{s}, O C C_{s}, O C C O_{2 s}$, and $P_{s}$, and $P_{S}$, obtained after simulating the operation model under study for the number of simulations (i.e., Iter $=270$ ) in Matrix $M_{p}$, as shown in Eqn (11) and presents the minimum and maximum of each performance metric:

$$
M_{p}=\left[\begin{array}{ccccc}
C_{1} & O C T_{1} & O C C_{1} & O C C O_{2_{1}} & P_{1} \\
C_{2} & O C T_{2} & O C C_{2} & O C C O_{2_{2}} & P_{2} \\
\vdots & \vdots & \vdots & \vdots & \vdots \\
C_{k} & O C T_{k} & O C C_{k} & O C C O_{2_{k}} & P_{k}
\end{array}\right] .
$$

The method checks if the current iteration counter $(k)$ is the same as the number of simulations (Iter) that was set either in Step 7, in the sensitivity analysis routine, or Step 21, in the stochastic simulation analysis routine, as shown in Eqn (10). If Eqn (12) is false, Steps 8-12 are repeated. Otherwise, Step 15 or Step 23 is executed for the sensitivity analysis routine or for the stochastic simulation analysis routine, respectively:

$$
k=\text { Iter? }
$$

The method checks if the current analysis method is sensitivity analysis or stochastic simulation and proceeds to either Step 15 or 23, respectively. It then queries the feasible solutions (resource combinations) that complete the operation within the user-defined ranges of performance metrics (i.e., $O C \mathrm{OC}_{2 u}, O C T_{u}$, and $O C C_{u}$ ) in Steps 15 and 16. It also identifies the optimal resource combination that satisfies the user-defined constraints $\left(S R_{S}\right)$ based on the performance metrics of all resource combinations $\left(R_{S}\right)$ using Eqn (13):

$$
\begin{aligned}
& S R_{S}=R_{S} ;\left(\forall R_{k} \in O C T_{k}>O C T_{u} \cap O C C_{k}>\right. \\
& \left.O C C_{u} \cap O C C O_{2 k}>O C C O_{2 u}\right),
\end{aligned}
$$

where: $S R_{S}$ is the satisfied results according to the constraints defined by the user; $R_{S}$ is the simulation results matrix generated by using all resource combinations; $O C T_{u}, O C C_{u}, O C C O_{2 u}$ is the job-site constraint of the operation completion time, cost, and carbon emission amount at the completion of an operation to feasible solutions, respectively.

If any of the performance metrics of a resource combination (i.e., $O C T_{k}, O C C_{k}, O C C O_{2 k}$ ) is greater than its corresponding constraint (i.e., $O C T_{u}, O C C_{u}$, and $\mathrm{OCCO}_{2 u}$ ), the resource combination is not a feasible solution. Any of the performance metrics can be used individually or jointly for parameter querying to identify the optimal resource combination from the set of feasible solutions satisfying the constraints $\left(S R_{S}\right)$ all at once in Steps 17 and 18. The feasible solutions may be re-sorted in ascending order by using a performance metric. Detailed simulation output data analysis may be performed on the sorted feasible solutions $\left(S R_{S}\right)$ in Step 19.

Finally, the method presents the sensitivity analysis results. The variability of the simulation output data (i.e., the cumulative amount of carbon emissions over the entire duration of operation, productivity, the amount of carbon emission at each work task component (i.e., NORMAL, COMBI, QUEUE), and the amount of carbon emission produced by each resource) obtained by assigning the optimal resource combination is presented in graphs.

\subsection{Executing stochastic simulation analysis}

The stochastic simulation analysis method starts by setting the analysis method variable to stochastic simulation (i.e., $M=S S$ ). The time measurement unit of the simulation system clock is unified in Step 21 by following the same process detailed in Step 6. Then, the method sets the number of simulation iterations to 120 by assuming a $99 \%$ confidence level and the iteration counter to zero (i.e., $k=0$ ) by default in Step 22. The eco-economic performance metrics computation process, which comprises Steps 8 to 13, is then performed. Because the variates of task durations are different in each simulation run, they create variable metrics (i.e., $C_{k}, O C T_{k}, O C C_{k}$, $O C C O_{2 k}$, and $P_{k}$ ). If the method detects that the current analysis method is stochastic simulation, it proceeds to Step 23 and computes the minimum number of simulations (MinIter) using the method championed by Ang and Tang (1975). The minimum number of simulations is calculated using the set of 120 OCTs. If the minimum number of simulations thus calculated is greater than the initial number of simulations (i.e., 120), as shown in Eqn (14), the simulation experiment does not pass the maturity test. The method replaces the initial number of simulations (Iter) with the minimum number of simulations (MinIter), invalidates the previous simulation process, and repeats Steps 8-23. Otherwise, it proceeds to Step 26 because the simulation experiment has passed the maturity test (Lee et al. 2010).

$$
\text { MinIter }<\text { Iter? }
$$

One hundred and twenty sets of simulation output data (i.e., $O C C O_{2 S}, O C T_{S}$, and $O C C_{S}$ ) are obtained and saved in matrix $M_{p}$. The best-fit-PDFs and their parameters of the performance metrics are computed using the automated best-fit-PDF algorithm (Lee et al. 2010) integrated into the $\mathrm{SCE}^{2}$ method. The method provides the best-fit-PDFs and their parameters of the perfor- 
mance metrics, their maximum and minimum ranges, the eco-economic performance metrics of each component (NORMAL, COMBI, and QUEUE) and those of each resource entity in Step 26. One who is equipped with the information given in Step 26 may query the probability to complete the construction operation under study within a user-defined eco-economic constraint of a performance metric (i.e., $O C C O_{2 l}, O C T_{l}$, and $O C C_{l}$ ). Finally, the $\mathrm{SCE}^{2}$ method computes the probability of completing the operation within the constraint and provides the information in a probability chart and table.

\section{Test case}

\subsection{Modelling a construction operation network}

The earthmoving operation model proposed by Halpin and Riggs (1992) was reproduced and modified to accommodate the "clean tires" work task to which labour entity is assigned as shown in Figure 2. It is used to demonstrate the computational procedure described in the preceding section, to illustrate the potential of $\mathrm{SCE}^{2}$ in the context of a small operation network that endured rigorous testing in earlier research, and to verify the validity of the $\mathrm{SCE}^{2}$ method. The earthmoving operation model comprises six queues (four resource queues and two idle queues), five work tasks ("load truck", "clean tires", "travel to dump", "spot and dump", and "return to load"), and one counter. It employs four types of resources, namely, "loader", "truck", "dozer", and "cleaning labour". More details on the queue, work task, and counter components of the earthmoving operation model are presented in Table 1.

\subsection{Initializing resource entities and setting termination rules}

The resource entities are initialized by specifying the minimum and maximum numbers of four resourcesspecifically, loader, cleaning labourer, dozer, and truck (e.g., $N_{L}=$ [1:3], $N_{C L}=$ [1:2], $N_{D}=$ [1:3], $N_{T}=$ [4:18]). The total number of resource combinations is $270(=$ $(3-1+1) \times(2-1+1) \times(3-1+1) \times(18-4+1))$. The unit of time used by the simulation clock was set to minute. The time delay function of each work task (i.e.,
COMBI or NORMAL) was defined by using the probability distribution function with the parameters shown in Table 1. The type of resource $\left(R_{T Y}\right)$ and resource state $\left(R_{S T}\right)$ (i.e., idle, lowly activated, medium activated, highly activated, and fully activated) were defined. Finally, the termination rules were specified by defining the maximum number of cycles $\left(N_{C Y}\right)$ and the $T_{L S}$ as 30 and infinity, respectively. Consequently, the simulation terminated when the production unit (e.g., empty truck after dump the earth) arrived at the counter 30 times.

\subsection{Executing sensitivity analysis}

Using the minimum and maximum numbers of resource entities initialized previously, sensitivity analyses were performed for 270 resource combinations. $\mathrm{SCE}^{2}$ identified the minimum and maximum of $\mathrm{OCCO}_{2}, O C T$, and $O C C$ of the operation as $[2634.83 ; 5339.77] \mathrm{kgCO}_{2}$, [132.88; 385.47] minute, and \$ [1922.32; 8605.59], respectively. Given the user-defined project constraints of $O C C O_{2 u}$ of $3000 \mathrm{kgCO}_{2}, O C T_{u}$ of 200 minutes, and $O C C_{u}$ of $\$ 2,500$, it was found that only 34 out of the 270 resource combinations satisfied these constraints. Out of these 34 feasible solutions, the global minimum OCT (132.88 minutes) was obtained when three loaders $\left(N_{L}=3\right)$, two cleaning labourers $\left(N_{C L}=2\right)$, one dozer $\left(N_{D}=1\right)$, and nine trucks $\left(N_{T}=9\right)$ were assigned to the earthmoving operation. In addition, the global minimum OCC $(\$ 1922.32)$ and $\mathrm{OCCO}_{2}\left(2634.83 \mathrm{kgCO}_{2}\right)$ were obtained when three loaders $\left(N_{L}=3\right)$, two cleaning labourers $\left(N_{C L}=2\right)$, one dozer $\left(N_{D}=1\right)$, and seven trucks $\left(N_{T}=7\right)$ were assigned to the operation.

The $\mathrm{SCE}^{2}$ method provides cumulative productivity over simulation time, cumulative carbon emission amount over simulation time, total carbon emission amount generated by a type of resource, and carbon emission amount generated at each work task and each queue component (i.e., NORMAL, COMBI, and QUEUE). It computes the eco-economic performance statistics, when the optimal resource combination (i.e., $N_{L}=3, N_{C L}=2$, $N_{D}=1$, and $N_{T}=7$ ) was assigned to the earthmoving operation. The amount of individual carbon emission $\left(\mathrm{kgCO}_{2 k}^{i}\right)$ of the loader, cleaning laborer, dozer, and

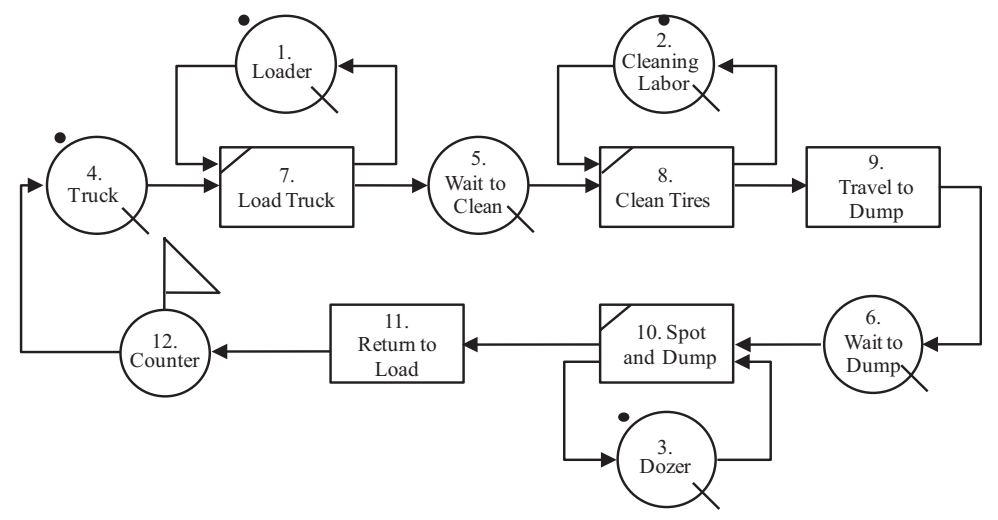

Fig. 2. Earthmoving operation model used as case study 
truck was $1399.35 \mathrm{kgCO}_{2}, 0.11 \mathrm{kgCO}, 127.72 \mathrm{kgCO}_{2}$, and $1107.65 \mathrm{kgCO}_{2}$, respectively. Therefore, the total amount of carbon emission $\left(\mathrm{OCCO}_{2 k}\right)$ of the operation under study was $2634.83 \mathrm{kgCO}$. This confirms that of all the resources the loader is the major source of carbon emission. This method encourages the achievement of optimal eco-economic resource combination by responsibly accommodating the priority changes required by job-site management.

\subsection{Executing stochastic simulation analysis}

Using the optimal resource combination obtained by using sensitivity analysis previously (i.e., three loaders, two cleaning labourers, one dozer, and seven trucks), stochastic simulation analysis is executed. The simulation output includes: (1) the productivity of the construction operation (i.e., the PDF of $O C T \mathrm{~s}$, and the PDF of the $O C C \mathrm{~s}$ ); (2) the eco-economic performance metrics of the construction operation (i.e., the PDF of the $\mathrm{OCCO}_{2} \mathrm{~s}$ ); (3) the total amount of carbon emission generated by work tasks and queue components; (4) the total amount of carbon emission generated by the operation; (5) the probability plot to complete the operation within a certain project specific requirement in performance metrics, as shown in Table 4. It confirms that the $\mathrm{SCE}^{2}$ method provides a stochastic eco-economic operation plan that can achieve low carbon and low-energy construction by using DES effectively.

Table 4. Experimental results obtained using $\mathrm{SCE}^{2}$

\begin{tabular}{ccccc}
\hline Performance & PDF & Parameters & Limit & Probability \\
\hline $\begin{array}{c}\text { Time } \\
\text { (minute) }\end{array}$ & Normal & $\begin{array}{c}\mathrm{mu}=146.33, \\
\text { sigma }=2.30\end{array}$ & 145 & $28.15 \%$ \\
\hline Cost(\$) & Normal & $\begin{array}{c}\mathrm{mu}=1980.35, \\
\text { sigma }=31.14\end{array}$ & 2000 & $73.60 \%$ \\
\hline $\begin{array}{c}\text { Emission } \\
(\mathrm{kgCO})\end{array}$ & G.E.V. & $\begin{array}{c}\mathrm{k}=-0.50, \\
\mathrm{mu}=2683.08, \\
\text { sigma }=46.37\end{array}$ & 2700 & $51.26 \%$ \\
\hline
\end{tabular}

\subsection{Comparison with existing system}

The earthmoving operation model proposed by Ahn (2012) shown in Figure 3 was reproduced and used to compare $\mathrm{SCE}^{2}$ and Ahn's method.

Total amount of the excavation is $60,095 \mathrm{yd}^{3}$ $\left(45,946 \mathrm{~m}^{3}\right)$ of topsoil, and the hauling distance is $1.6 \mathrm{~km}$. The major resources involved in the model are loaders, trucks, and earth. The resource matrix defining the resource entities and work tasks of the model is presented in Table 5. The model has five work tasks (i.e., "exchanging truck", "loading", "hauling", "dumping", and "returning") and two queues (i.e., "Truck" and "Loader"). A loader traverse "exchanging truck" and "loading" tasks and waits in "loader" queue. It is "idle" state in "Loader" queue and "exchanging truck" task. However, it is "Highly accelerated" state in "Loading" task. A truck traverses "exchanging truck", "loading", "hauling", "Dumping", and "Returning" tasks and waits in "truck" queue. It is "idle" in "Truck" queue, "Lowly activated" in "Exchanging truck" task, "idle" in "Loading" task, "Highly activated" in "Hauling" task, "Lowly activated" in "Dumping" tasks, and "Medium activated" in "Returning" task.

Ahn's method (2012) computes 5,300 $\mathrm{kgCO}_{2}$ from non-valuable operation of equipments (i.e., resources' idle state) and $36,300 \mathrm{kgCO}_{2}$ from valuable operation of equipments (i.e., resources' active state). However, $\mathrm{SCE}^{2}$ computes $7,247 \mathrm{kgCO}_{2}$ from non-valuable operations of equipments and $43,332.85 \mathrm{kgCO}_{2}$ from valuable operations of equipments when the transitory nature of the resources states was considered. The difference of $\mathrm{CO}_{2}$ amounts generated from non-valuable operations of equipments using the two methods is $1,947 \mathrm{kgCO}_{2}$ $(=7,247-5,300)$. It is attributed to the fact that Ahn's method (2012) assumes the average hourly fuel consumption in idle state is $20 \%$ of the average hourly fuel consumption in active state according to Lewis et al. (2011), while $\mathrm{SCE}^{2}$ assumes the average hourly fuel consumption in idle state is $10 \%$ of the average hourly fuel consumption in fully accelerated state of the resources (Caterpillar Inc. 2010). That is, Ahn (2012) assumes that a loader's and a truck's average hourly fuel consumption in idle state are $9.71 /$ hour $(=20 \%$ of $48.51 /$ hour $)$ and $5.56 \mathrm{l} /$ hour ( $=20 \%$ of $27.8 \mathrm{l} /$ hour), respectively. However, $\mathrm{SCE}^{2}$ considers that a loader's and a truck's average hourly fuel consumption in idle state are $13.84 \mathrm{l} /$ hour $(=10 \%$ of $138.41 /$ hour $)$ and $7.971 /$ hour $(=10 \%$ of 79.7 1/hour), respectively. In addition, when $\mathrm{SCE}^{2}$ was run using the Ahn's operation model (Fig. 3) and $\mathrm{SCE}^{2}$ 's assumptions that classifies the resource states into the full range of possible states (i.e., 5 categories) while holding Ahn's assumption that the average hourly fuel consumption in idle state is $20 \%$ of the average hourly fuel consumption in active state, the $\mathrm{CO}_{2}$ amounts generated from non-valuable operations of equipments and from valuable operations of equipments were around 5,300 $\mathrm{kgCO}_{2}$ and $43,333 \mathrm{kgCO}_{2}$, respectively. This is proof that $\mathrm{SCE}^{2}$ capture the transitory nature of the resources states more expeditiously and reliably than the existing method, and is a testament to the reliability of $\mathrm{SCE}^{2}$. While $\mathrm{SCE}^{2}$ is conservative because it systematically overestimates the average hourly fuel consumption in idle state, $\mathrm{SCE}^{2}$

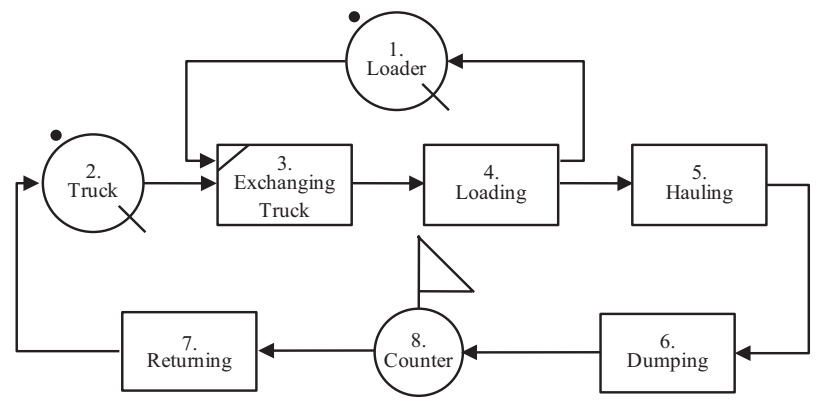

Fig. 3. Earthmoving operation model (adapted from Ahn 2012) 
Table 5. Resource matrix defining the resource entities and work tasks for test case 2

\begin{tabular}{|c|c|c|c|c|c|c|c|c|c|c|c|c|}
\hline \multicolumn{3}{|c|}{ Components } & \multicolumn{10}{|c|}{ Resources } \\
\hline Type & ID & Name & $W_{I D}$ & $R_{I D}$ & $R_{T Y}$ & $R_{S T}$ & $\begin{array}{l}\text { Model } \\
\left(R_{M}\right)\end{array}$ & $\begin{array}{l}\text { Engine } \\
\text { Type } \\
\left(E T_{i}\right)\end{array}$ & $\begin{array}{l}\text { Average fuel } \\
\text { consumption } \\
\text { (1/hour) }\end{array}$ & $\begin{array}{c}\text { No. of resource }\left(N_{i}\right) \\
\text { Or } \\
\text { Time delay } \\
\text { functions }\end{array}$ & Pre. & Suc. \\
\hline \multirow{2}{*}{ Queue } & 1 & Loader & 1 & 1 & $E$ & I & Komatsu EX750 & Diesel & 13.84 & 2 & 4 & 3 \\
\hline & 2 & Truck & 2 & 2 & $\mathrm{E}$ & I & Caterpillar 740 & Diesel & 7.97 & 11 & 7 & 3 \\
\hline \multirow{7}{*}{$\begin{array}{l}\text { Work } \\
\text { Task }\end{array}$} & \multirow{2}{*}{3} & \multirow{2}{*}{$\begin{array}{l}\text { Exchanging } \\
\text { Truck }\end{array}$} & 3 & 1 & $\mathrm{E}$ & I & Komatsu EX750 & Diesel & 13.84 & \multirow{2}{*}{ - Uniform $(0.90,1.10)$} & \multirow{2}{*}{1,2} & \multirow{2}{*}{4} \\
\hline & & & 4 & 2 & $\mathrm{E}$ & $\mathrm{L}$ & Caterpillar 740 & Diesel & 19.95 & & & \\
\hline & \multirow{2}{*}{4} & \multirow{2}{*}{ Loading } & 5 & 1 & $\mathrm{E}$ & $\mathrm{H}$ & Komatsu EX750 & Diesel & 62.29 & \multirow{2}{*}{ Uniform $(1.50,3.00)$} & \multirow{2}{*}{3} & \multirow{2}{*}{1,5} \\
\hline & & & 6 & 2 & $\mathrm{E}$ & $\mathrm{I}$ & Caterpillar 740 & Diesel & 7.97 & & & \\
\hline & 5 & Hauling & 7 & 2 & $\mathrm{E}$ & $\mathrm{H}$ & Caterpillar 740 & Diesel & 39.85 & Uniform $(3.00,7.50)$ & 4 & 6 \\
\hline & 6 & Dumping & 8 & 2 & $\mathrm{E}$ & $\mathrm{L}$ & Caterpillar 740 & Diesel & 19.95 & Uniform $(0.75,2.25)$ & 5 & 8 \\
\hline & 7 & Returning & 9 & 2 & $\mathrm{E}$ & $\mathrm{M}$ & Caterpillar 740 & Diesel & 27.90 & Uniform $(1.32,3.08)$ & 8 & 2 \\
\hline Counter & 8 & Counter & - & - & - & - & - & - & - & - & 6 & 7 \\
\hline
\end{tabular}

enhances practicality by handling large operation model with many stochastic work tasks quickly and reach more viable and realistic solution. Noteworthy is that the existing methods do not provide a mean to specify the full range of possible resource states, but do make use of the amount of times obtained from commercial systems that define resource state either "active" or "idle" state only by default. $\mathrm{SCE}^{2}$ increases the prediction accuracy by taking into account the transitory resource states that change according to its operating conditions in a specific node and handle the variability of the carbon emission amount more effectively than existing methods. The improved performance justifies the academic and practical contributions of the mathematical formulation and method.

\subsection{Benefits and limitations of using the stochastic carbon emission estimation method ( $\left.\mathrm{SCE}^{2}\right)$}

The benefits of using $\mathrm{SCE}^{2}$ can be summarized as follows: First, $\mathrm{SCE}^{2}$ builds upon a well-established system with which practitioners are already familiar and with standard compatibility with industry foundation (e.g., SimEvent 2012). Second, $\mathrm{SCE}^{2}$ expeditiously generates the stochastic eco-economic performance metrics if four pieces of information are provided: (1) operation model; (2) the set of resource attributes and the resource state; (3) equipment and labourer database; and (4) stopping rules. Third, it provides a multi-objective optimization method that finds the optimal $\mathrm{OCCO}_{2}-\mathrm{OCT}-\mathrm{OCC}$ tradeoff, which identifies an optimal resource combination for a construction operation.

The limitations of the $\mathrm{SCE}^{2}$ method are as follows: First, the equipment performance data used in this study is limited to the information provided by Caterpillar Inc. (2010). Second, the hourly average fuel consumptions in idle and fully accelerated operating conditions are obtained by extrapolating three values relative to an engine's operating status (i.e., low, medium, and high) given by Caterpillar Inc. (2010). It would be desirable to collect them from actual measurements to calibrate the accuracy of the method. Finally, $\mathrm{SCE}^{2}$ takes into account only the carbon emissions at the operation level. It would be more appealing if $\mathrm{SCE}^{2}$ could be integrated with project management software at the project level.

\section{Results and conclusion}

The main contribution of this study is the development of a stochastic carbon emission estimation method for construction operations using DES. The contribution also includes the development of an easy-to-use computerized tool called $\mathrm{SCE}^{2}$ that efficiently implements this method in a system that is most appropriate for the simulation of construction operation networks. Further details of the contributions included with the method are as follows; first, it estimates the variability of the amount of carbon emission generated either by all resources jointly or by a resource individually. Second, whereas existing DESbased methods classify resource states into either "active" or "idle" only; the $\mathrm{SCE}^{2}$ method takes into account the transitory resource states that change according to its operating conditions at different locations in the model (i.e., resource queue, idle queue, and work tasks). It facilitates modelling of the transitory nature of resource states by defining them in five categories (namely, idle, lowly activated, medium activated, highly activated, and fully accelerated) in a specific event, and handle the variability of the carbon emission amount more effectively than existing methods. Third, it provides a multi-objective optimization method that effectively identifies the optimal resource combination by considering multiple performance metrics (specifically, $\mathrm{OCCO}_{2}, O C T$, and/or $O C C$ ) all at once. It searches for the optimal resource combination that meets job-site-specific constraints and estimates the best-fit-PDFs of $O C C_{2} \mathrm{~s}, O C T \mathrm{~s}$, and $O C C \mathrm{~s}$ for each resource entity, work task component, and the entire operation. Finally, $\mathrm{SCE}^{2}$ facilitates making more informed 
decisions because it allows very rapid modelling and analysis of the eco-economic risk of an operation by integrating the existing simulation system and stochastic carbon emission entities. In summary, it advances the ongoing research and the body of knowledge in eco-economic operation modelling and analysis because it helps project managers to achieve project goals while honouring the predefined constraints associated with carbon emission, time, and budget.

\section{Acknowledgements}

This work was supported by the National Research Foundation of Korea (NRF) grant funded by the Korea government (MSIP) (No. 2013R1A2A2A01068316). The contribution of the Ministry of Science, ICT and Future Planning is gratefully acknowledged.

\section{References}

Ahn, C. B. 2012. An integrated framework for sustainable construction processes: understanding and managing the environmental performance of construction operations: $\mathrm{PhD}$ dissertation. University of Urbana, Illinois, USA.

Ahn, C. B.; Lee, S. H. 2012. Importance of operational efficiency to achieve energy efficiency and exhaust emission reduction of construction operations, Journal of Construction Engineering and Management 139(4): 404-413. http://dx.doi.org/10.1061/(ASCE)CO.1943-7862.0000609

Ahn, C. B.; Lee, S. H.; Pena-Mora, F. A. 2010. Enhanced estimation of air emissions from construction operations based on discrete-event simulation, in Proc. of the International Conference on Computing in Civil and Building Engineering (ICCCBE), 2010, Nottingham, UK. 7 p.

Ang, A. H.-S.; Tang, W. H. 1975. Probability concepts in engineering planning and design. Volume I-basic principles. New York, NY: Wiley. 424 p.

Cass, D.; Mukherjee, A. 2011. Calculation of greenhouse gas emissions for highway construction operations by using a hybrid life-cycle assessment approach: Case study for pavement operations, Journal of Construction Engineering and Management 137(11): 1015-1025. http://dx.doi.org/10.1061/(ASCE)CO.1943-7862.0000349

Caterpillar Inc. 2010. Caterpillar performance handbook. $40^{\text {th }}$ ed. Caterpillar. Peoria, IL. 1014 p.

EPA. 2009. Potential for reducing greenhouse gas emissions in the construction sector. US EPA, Washington, DC, USA [online], [cited 13 October 2012]. Available from Internet: http://www.epa.gov/sectors/pdf/construction-sectorreport.pdf

Gonzalez, V.; Echaveguren, T. 2012. Exploring the environmental modeling of road construction operations using discrete-event simulation, Automation in Construction 24: 100-110. http://dx.doi.org/10.1016/j.autcon.2012.02.011

Halpin, D. W.; Riggs, L. S. 1992. Planning and analysis of construction operations. New York, NY: John Wiley \& Sons. $400 \mathrm{p}$.

Hasan, S.; Bouferguene, A.; Al-Hussein, M.; Gillis, P.; Telyas, A. 2013. Productivity and $\mathrm{CO}_{2}$ emission analysis for tower crane utilization in high-rise building projects, $A u$ tomation in Construction 31: 255-264.

http://dx.doi.org/10.1016/j.autcon.2012.11.044
Int Panis, L.; De Geus, B.; Vandenbulcke, G.; Willems, H.; Degraeuwe, B.; Bleux, N.; Mishra, V.; Thomas, I.; Meeusen, R. 2010. Exposure to particulate matter in traffic: a comparison of cyclists and car passengers, Atmospheric Environment 44(19): 2263-2270.

http://dx.doi.org/10.1016/j.atmosenv.2010.04.028

IPCC. 2006. IPCC guidelines for national greenhouse gas inventories. IGES, Hayama, Japan. 1200 p.

Kelton, W. D.; Law, A. M. 2000. Simulation modeling and analysis. Boston, MA: McGraw Hill. 804 p.

Lee, D. E.; Yi, C. Y.; Lim, T. K.; Arditi, D. 2010. Integrated simulation system for construction operation and project scheduling, Journal of Computing in Civil Engineering 24(6): 557-569. http://dx.doi.org/10.1061/(ASCE)CP.1943-5487.0000061

Lewis, P.; Leming, M.; Rasdorf, W. 2011. Impact of engine idling on fuel use and $\mathrm{CO}_{2}$ emissions of nonroad diesel construction equipment, Journal of Management in Engineering 28(1): 31-38. http://dx.doi.org/10.1061/(ASCE)ME.1943-5479.0000068

Liu, S.; Tao, R.; Tam, C. M. 2013. Optimizing cost and $\mathrm{CO}_{2}$ emission for construction projects using particle swarm optimization, Habitat International 37: 155-162. http://dx.doi.org/10.1016/j.habitatint.2011.12.012

Li, X. D.; Zhu, Y. M.; Zhang, Z. H. 2010. An LCA-based environmental impact assessment model for construction process, Building and Environment 45(3): 766-775. http://dx.doi.org/10.1016/j.buildenv.2009.08.010

Peurifoy, R. L.; Schexnayder, C. J.; Shapira, A. 2009. Construction planning, equipment, and methods. New York, NY: Mcgraw-Hill. $832 \mathrm{p}$.

Ragheb, A. F. 2011. Towards environmental profiling for office buildings using Life Cycle Assessment (LCA): $\mathrm{PhD}$ dissertation. University of Michigan, Michigan, USA.

Sharrard, A. L.; Matthews, H. S.; Ries, R. J. 2008. Estimating construction project environmental effects using an inputoutput-based hybrid life-cycle assessment model, Journal of Infrastructure Systems 14(4): 327-336.

http://dx.doi.org/10.1061/(ASCE)1076-0342(2008)14:4(327)

Sherwood, L. 2006. Fundamentals of physiology with infotrac: a human perspective. Cengage Learning, UK. 768 p.

SimEvent. 2012. Operations research and optimization of discrete event simulation [online], [cited 10 October 2012]. Available from Internet: http://www.mathworks.com/videos

Tae, S. H.; Shin, S.W.; Woo, J. H.; Roh, S. J. 2011. The development of apartment house life cycle $\mathrm{CO}_{2}$ simple assessment system using standard apartment house of South Korea, Renewable and Sustainable Energy Reviews 15(3): 1454-1467. http://dx.doi.org/10.1016/j.rser.2010.09.053

Vieira, P. M. 2007. Environmental assessment of office buildings: $\mathrm{PhD}$ dissertation. University of California, Berkeley, USA.

Yan, H.; Shen, Q.; Fan, L. C. H.; Wang, Y.; Zhang, L. 2010. Greenhouse gas emissions in building construction: a case study of One Peking in Hong Kong, Building and Environment 45(4): 949-955. http://dx.doi.org/10.1016/j.buildenv.2009.09.014

Zapata, P.; Gambatese, J. A. 2005. Energy consumption of asphalt and reinforced concrete pavement materials and construction, Journal of Infrastructure Systems 11(1): 9-20. http://dx.doi.org/10.1061/(ASCE)1076-0342(2005)11:1(9) 
Chang-Yong YI. Mr. Yi is a PhD Candidate under supervision of Professor. Dong-eun Lee in the school of architecture and civil engineering at Kyungpook National University. He is currently working on a research project whose objective is to develop construction multi objective eco-time-quality-cost optimization system using stochastic simulation techniques.

Han-Seong GWAK. Mr. Gwak is a graduate student under supervision of Professor. Dong-Eun Lee in the school of architecture and civil engineering at Kyungpook National University. He is currently working on a research project whose objective is to develop eco-economic decision making system by hybridizing optimization algorithm and simulation techniques.

Dong-Eun LEE. Dr Lee had worked for School of Engineering at Southern Illinois University Edwardsville (SIUE) in USA as an assistant professor. He is a tenured professor in the school of architecture and civil engineering at Kyungpook National University in South Korea. Member of American Society of Civil Engineers (ASCE), Korean Society of Civil Engineers (KSCE), Korea Institute of Construction Engineering and Management (KICEM), and Architectural Institute of Korea, etc. His research interests include (1) Simulation based scheduling method; (2) Stochastic simulation and Quantitative analysis; (3) Construction business process modeling and automation; (4) Workflow Management Systems (WfMS) in construction; (5) Construction quality and safety. Dr Lee's major research effort has been focusing on developing and employing simulation techniques for resolving issues in construction. His research interest also includes Expert system and general computer applications in construction. 